\title{
FRACTURE RESISTANCE OF VARIOUS BULK FILL COMPOSITE RESIN IN ENDODONTICALLY TREATED CLASS I PREMOLAR (AN IN-VITRO STUDY)
}

\author{
Wandania Farahanny1, Dennis ${ }^{2}$, Monica Dashni Aruldas ${ }^{3}$ \\ ${ }^{1}$ Faculty of Dentistry, University of Sumatera, Utara. \\ ${ }^{2}$ Faculty of Dentistry, University of Sumatera, Utara. \\ ${ }^{3}$ Faculty of Dentistry, University of Sumatera, Utara.
}

ABSTRACT

\section{BACKGROUND}

Endodontically treated teeth are more susceptible to fracture as a result of increased brittleness and are weakened because of coronal destruction. Endodontically treated teeth with small or minimal cavities can be restored with direct composites in the presence of buccal and palatal cusp with intact marginal ridge. Bulk fill has minimal polymerisation shrinkage rate, curing depth of $4 \mathrm{~mm}$, better bond strength and good physical properties. Other advantages include fewer void in the restorative material and lesser risk of contamination between layers.

\section{MATERIALS AND METHODS}

Forty intact human upper premolars were selected for this study. In G1 to G4 standard class I cavity preparation with mesiodistal length of $4 \mathrm{~mm}$ and bucco-palatal width of $3 \mathrm{~mm}$ were done. A conservative endodontic access was done. All canals were prepared with Ni-Ti K files using a step-back technique. All teeth were obturated with gutta-percha. The teeth were randomly divided into four groups, G1 Tetric N Ceram bulk fill, G2 Filtek bulk fill, G3 Smart Dentin Replacement bulk fill and G4 Packable composite Filtek P60.

\section{RESULTS}

There was a statistically significant effect on the fracture resistance of endodontically treated premolars with class I cavities using bulk fill composites $(\mathrm{p}<0.05)$.

\section{CONCLUSION}

Bulk fill composite can be used as a final restoration in endodontically treated premolars with class I cavity, as it can also preserve sound tooth structure which can increase fracture resistance of the tooth.

\section{KEYWORDS}

Bulk Fill Composite, Endodontically Treated Teeth, Fracture Resistance.

HOW TO CITE THIS ARTICLE: Farahanny W, Dennis, Aruldas MD. Fracture resistance of various bulk fill composite resin in endodontically treated class I premolar (an in-vitro study). J. Evolution Med. Dent. Sci. 2017;6(72):5168-5171, DOI: $10.14260 /$ Jemds/2017/1122

\section{BACKGROUND}

Endodontic treatment is done by eliminating the inflamed or infected pulpal tissue. Endodontically treated teeth are more susceptible to fracture as a result of increased brittleness and are weakened because of coronal destruction from dental caries, access cavity preparation, instrumentation of the root canal, previous fracture, loss of moisture in the dentin and previous restorations or endodontic techniques. Excess loss of tooth structure will cause the teeth to reduce in strength. ${ }^{1}$ Therefore, endodontically treated teeth are at a higher risk of fracture than vital teeth. ${ }^{1}$

The ability of the tooth to resist occlusal forces is directly related to the amount of remaining coronal tooth structure. ${ }^{1}$ Maxillary premolars have an anatomic shape that makes them more susceptible to cusp fractures when under occlusal load. ${ }^{1}$ Posterior teeth receive significantly greater masticatory forces than the anterior teeth. ${ }^{2}$

Financial or Other, Competing Interest: None.

Submission 21-02-2017, Peer Review 23-08-2017,

Acceptance 30-08-2017, Published 07-09-2017.

Corresponding Author:

Dennis,

JL. Alumni No. 2,

Kampus USU, Medan-20155.

E-mail: wanda_sidentist@yahoo.com

DOI: $10.14260 /$ jemds $/ 2017 / 1122$
Therefore, a restorative material which can receive large occlusal forces is required.

One of the most common restorations used is a composite resin, adhesion of the composite to the cavity wall will reinforce the remaining tooth structure and will increase its resistance against fracture. ${ }^{2}$

In general, non-vital teeth are usually restored using a post and core system, full crown or inlay that protects the cusps of the teeth, but recent technology developments in the field of restorative dentistry such as the increase in physical properties of composites and the use of adhesive system provides new potential in restoring a non-vital tooth. ${ }^{3}$ Based on the concept of minimally invasive dentistry, the use of direct composites help preserve tooth structure as much as possible. ${ }^{4}$ Meanwhile, the use of posts reduces fracture resistance of endodontically treated teeth because the removal of radicular dentin during root canal preparation to accommodate the post would weaken the teeth and occlusal load can be channelled through intra-radicular means by the posts, thereby increasing the incidence of vertical root fracture.5,6 Teeth with small or minimal cavities can be restored with direct composites in the presence of buccal and palatal cusp with intact marginal ridge. ${ }^{7}$

Composite restorations are a good alternative for posterior teeth; however, a disadvantage of composite resins is polymerisation shrinkage. Internal contraction stress 
causes the composite to move away from the cavity wall, which can damage the marginal seal of the restoration and cause microleakage. Microleakage on the other hand causes deformation of the tooth structure, which leads to microcrack and eventually causes fracture. ${ }^{8}$ Incremental technique is usually used in order to overcome this, but this technique requires special handling, more time and skills because the composite is applied layer by layer. Therefore, a new restorative material known as bulk fill resin based composites (RBSs), which can be applied in a single layer was introduced. ${ }^{9}$

Bulk fill composites have certain characteristics such as minimal polymerisation shrinkage rate, curing depth of 4 $\mathrm{mm}$, better bond strength compared to conventional composites and good physical properties. ${ }^{9}$ Other advantages of bulk filling technique include fewer void in the restorative material and lesser risk of contamination between layers. ${ }^{10}$ Each type of bulk fill has its own mechanism and advantages of its own. Damanhoury and Platt (2014), in their study about shrinkage in bulk fill found that it has a curing efficiency of $80 \%$, a lower stress shrinkage compared to incrementally filled composites and has potential to be used in posterior teeth. ${ }^{10}$ The aim of this study is to investigate the effect of bulk fill composites on endodontically treated premolars with class I cavity.

\section{MATERIALS AND METHODS}

In this laboratory experimental study, the study design was Posttest Only Control Group. Human maxillary premolars were obtained in accordance with the ethical guidelines of the North Sumatera University Ethics Committee. The selection criteria were fresh intact individual human maxillary premolars, caries free without any restorations that were extracted for orthodontic purposes with a range of maximum buccal-palatal-widths (BPWs) varying from 8.4 to $8.8 \mathrm{~mm}$ when measured with a calliper were selected for this study. All calculus deposits were removed by hand-scaling. After being stored in saline solution at room temperature, teeth surfaces were cleaned with pumice and water; 40 premolars were divided in four groups with ten teeth in each group.

In G1 to G4 standard class I cavity preparation with mesio-distal length of $4 \mathrm{~mm}$ and bucco-palatal width of $3 \mathrm{~mm}$ were done. A periodontal probe was used to measure cavity dimensions. Cavity preparation was done using a high-speed hand piece and under air-water spray with an endo access bur. A conservative endodontic access was done on the pulp chamber wall. Next, all canals were prepared with Ni-Ti K files using a step-back technique. Initially, the canal is widened by 3 ISO sizes, then the working length is reduced by $1.0 \mathrm{~mm}$ and by alternating a reduction and the master file the preparation is completed. Sodium hypochlorite (2.5\%) solution was used during root canal cleaning and shaping, while chlorhexidine solution was used as final flush.

After instrumentation, all teeth were obturated with gutta-percha (Dochem, UK) and endodontic sealer (MasterDent, USA) by the lateral condensation technique. After obturation gutta-percha was removed to a depth of $2 \mathrm{~mm}$ from the canals with Gates-Glidden drill for retention purposes. RMGIC (GC Gold Label, Japan) was applied as an intra-orifice barrier.
The Teeth were randomly divided into Four Groups (10 Teeth in each group) according to the type of the Restorative Material that was used-

Group 1: Endodontic treatment with class I cavity, restored with Tetric N Ceram bulk fill (Ivoclar, Liechtenstein).

Group 2: Endodontic treatment with class I cavity, restored with Filtek bulk fill (3M ESPE, USA).

Group 3: Endodontic treatment with class I cavity, restored with Smart Dentin Replacement bulk fill (Dentsply, USA).

Group 4: Endodontic treatment with class I cavity, restored with Packable composite Filtek P60 (3M ESPE, USA) (control group).

All teeth were etched with $37 \%$ phosphoric acid for 15 seconds and washed with water for 15 seconds. A one-bottle adhesive system was then applied to the cavities with a microbrush according to the manufacturer's instructions and photopolymerised for 20 seconds. Each composite was applied in a bulk technique up to $4 \mathrm{~mm}$ except for the G3 and G4. Smart Dentin Replacement bulk fill, G3 was applied as an intermediate layer in a bulk technique upto $3 \mathrm{~mm}$ and was cured for 20 seconds. A layer of packable composite with 2 mm thickness was applied over it and cured for 20 seconds. In the control group, the packable composite was applied in an incremental technique and each layer was cured for 20 seconds. All the teeth were polished using fine and extra fine finishing bur.

All the samples were then stored in saline solution for 24 hours. Thermocycling process was done by first soaking the samples in a beaker glass filled with iced water $\left(5^{\circ} \mathrm{C}\right)$ for 30 seconds, then transferring it with the interval of 10 seconds to a waterbath of $55^{\circ} \mathrm{C}$ for another 30 seconds. This process was repeated 200 times.

A self-cured acrylic resin cylinder was used to fix each tooth at a distance of $2.0 \mathrm{~mm}$ from the cementum enamel junction. The samples were subjected to a compressive load at a crosshead speed of $1 \mathrm{~mm} /$ minute using Torsee's Electronic System Universal Testing Machine. Compressive loading was applied using a custom made metal jig placed in the centre of the tooth. The force required to cause fracture (Kgf) was recorded and converted to Newton (N), $1 \mathrm{Kgf}=9,8$ $\mathrm{N}$, and the results were submitted to statistical analysis by one-way ANOVA and Post Hoc test ( $p<0.05)$ using the SPSS Statistics version 22 software.

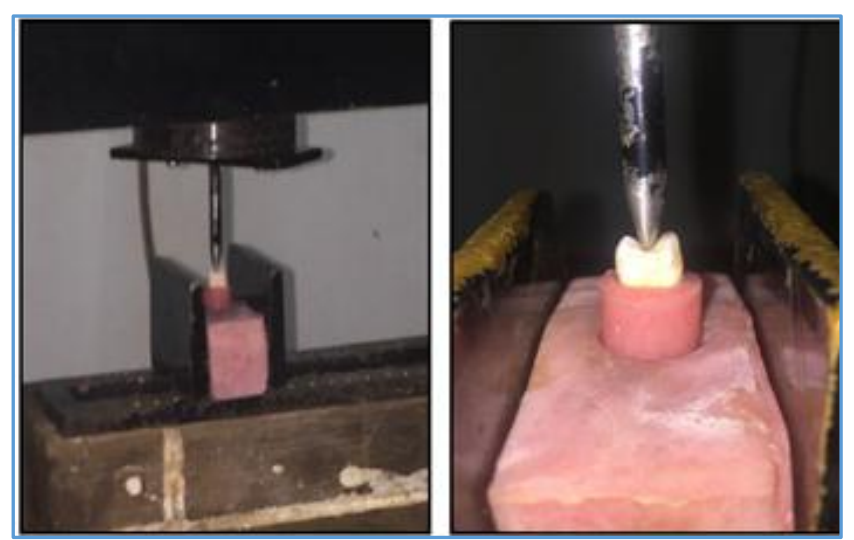

Figure 1. Fracture Test using Torsee's Universal Testing Machine 
One-way ANOVA was used to analyse the difference of fracture resistance between each group $(\alpha=0.05)$. The highest fracture resistance was recorded in G2, Filtek bulk fill with the value of $982.1 \mathrm{~N}$ and standard deviation of 157.9. Group 1, Tetric N Ceram bulk fill had an average of $832.2 \mathrm{~N}$, whereas G3 SDR bulk fill composite was $669.8 \mathrm{~N}$ and lastly G4 had the lowest fracture resistance with an average of $613.9 \mathrm{~N}$. The average and standard deviation of the fracture resistance of each group is shown in Table 1 . Table 2 on the other hand shows the difference in the average and the $p$ value of each group.

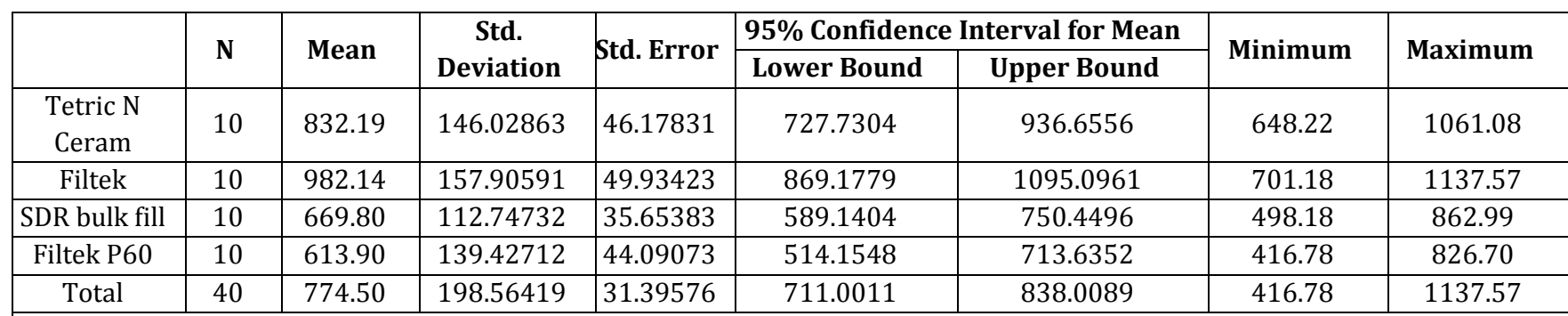

\section{Table 1. Descriptive Data showing the Mean and Standard Deviation of Fracture Resistance using One-Way ANOVA Test}

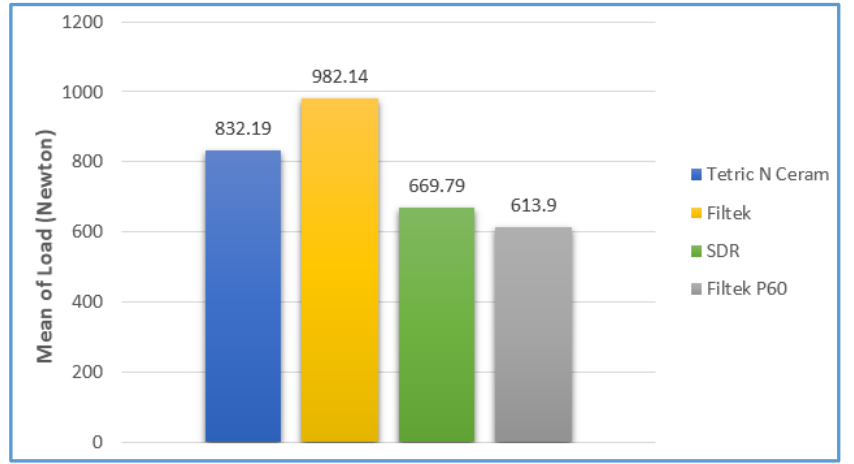

Figure 2. Graphic showing the Average Load Value of Tetric N Ceram Bulk Fill, Filtek Bulk Fill, SDR Bulk Fill and Packable Composite

\begin{tabular}{|c|c|c|c|c|c|c|}
\hline \multirow{2}{*}{$\begin{array}{c}\text { (I) } \\
\text { Group }\end{array}$} & \multirow{2}{*}{ (J) Group } & \multirow{2}{*}{ Mean Difference (I-J) } & \multirow{2}{*}{ Std. Error } & \multirow{2}{*}{ Sig. } & \multicolumn{2}{|c|}{ 95\% Confidence Interval } \\
\hline & & & & & Lower Bound & Upper Bound \\
\hline \multirow{3}{*}{ G I } & G II & $-149.94400^{*}$ & 62.61410 & .022 & -276.9313 & -22.9567 \\
\hline & G III & $162.39800^{*}$ & 62.61410 & .014 & 35.4107 & 289.3853 \\
\hline & G IV & $218.29800^{*}$ & 62.61410 & .001 & 91.3107 & 345.2853 \\
\hline \multirow{3}{*}{ G II } & G I & $149.94400^{*}$ & 62.61410 & .022 & 22.9567 & 276.9313 \\
\hline & G III & $312.34200^{*}$ & 62.61410 & .0001 & 185.3547 & 439.3293 \\
\hline & G IV & $368.24200^{*}$ & 62.61410 & .0001 & 241.2547 & 495.2293 \\
\hline \multirow{3}{*}{ G III } & G I & $-162.39800^{*}$ & 62.61410 & .014 & -289.3853 & -35.4107 \\
\hline & G II & $-312.34200^{*}$ & 62.61410 & .0001 & -439.3293 & -185.3547 \\
\hline & G IV & 55.90000 & 62.61410 & .378 & -71.0873 & 182.8873 \\
\hline \multirow{3}{*}{ G IV } & G I & $-218.29800^{*}$ & 62.61410 & .001 & -345.2853 & -91.3107 \\
\hline & G II & $-368.24200^{*}$ & 62.61410 & .0001 & -495.2293 & -241.2547 \\
\hline & G III & -55.90000 & 62.61410 & .378 & -182.8873 & 71.0873 \\
\hline
\end{tabular}

Table 2. Post Hoc Test Result shows the Difference in the Average and P Value of the Fracture Resistance in Each Group

*. The mean difference is significant at the 0.05 level.

\section{DISCUSSION}

In this study, maxillary premolars were used as samples with several criteria such as tooth length and the crown size which did not differ much, no crown fracture, unrestored, intact crown and no caries. Studies have shown that endodontic treatment is one of the main aetiologies for tooth fracture. This is mainly because of the biomechanical and neuroreseptive changes in the teeth due to the loss of pulpal tissue which will cause dehydration, demineralisation, changes in collagen and reduced levels of proprioception which can impair normal protective reflexes of the pulp.
Post endodontic restoration is a very important aspect in the success of a endodontically treated tooth. The opinion of dentist in general is to restore an endodotically treated tooth with indirect restoration using post, core and a full crown, but in recent years there are studies that suggest the use of direct restoration technique without the need of a post because adhesive restorations can increase cusp stiffness and does not require cuspal coverage. Moreover, direct restorations enable us to preserve the tooth structure, where else post placement will only cause more structural loss which can affect the teeth's resistance towards fracture.

The results show that Group 2, which is restored with Filtek bulk fill has the highest average fracture resistance of 
982.1 N when compared to Tetric N Ceram bulk fill with an average of $832.2 \mathrm{~N}$, SDR bulk fill with an average of $669.8 \mathrm{~N}$ and packable composite with an average of $613.9 \mathrm{~N}$. Therefore, the statistical analysis proves that the use of bulk fill composite in endodontically treated maxillary premolar with class I cavity influences the fracture resistance of the teeth.

Bulk fill is a new composite that aims to reduce restorative procedures by allowing application of the composite upto $4 \mathrm{~mm}$ thickness and eventually reducing the porosity and achieving a better restoration consistency. In this study, bulk fill composites used were expected to increase the fracture resistance of the teeth post endodontic treatment in order to withstand occlusal forces which are dynamic in nature.

In this study, the fracture load of teeth in Group 2 with Filtek bulk fill restoration ( $982.1 \mathrm{~N}$ ) is higher than of the ones with Tetric $\mathrm{N}$ Ceram bulk fill restoration (832.2 N). This can be explained by the higher filler content in Filtek bulk fill. In addition, Filtek bulk fill has silica/zirconia cluster fillers which are known as nanoclusters with the average filler size of $20 \mathrm{~nm}$ for silica and $4-11 \mathrm{~nm}$ for zirconia. Nanocluster particles have better mechanical properties compared to spheroidal or irregularly shaped fillers, in which case the use of nanocluster particles in a composite can modify the failure mechanism and increase the resistance of the material. ${ }^{11}$ Besides that Filtek bulk fill has a higher compressive strength of $300 \mathrm{MPa}$, whereas Tetric $\mathrm{N}$ Ceram bulk fill has a compressive strength of $224 \mathrm{MPa} .12$

Fracture load of teeth in Group 1 with Tetric N Ceram bulk fill restoration is higher than of the ones with SDR bulk fill restoration. This might be due to the presence of a filler with low elastic modulus in Tetric N Ceram bulk fill, which acts as a shrinkage stress reliever and also a new light initiator known as Ivocerin, which can absorb blue light wavelengths ranging from $370-460 \mathrm{~nm}$. Based on the product profile of Tetric N Ceram bulk fill, Ivocerin is said to be more reactive towards light compared to camphorquinone, which makes polymerisation faster with a deeper curing depth. ${ }^{12}$ Previously, photoinitiators that are most commonly used include Camphorquinone (CQ), phenylpropanedione (PPD), mono- or bis-acylphosphine oxides (MAPO and BAPO), benzoyl germanium and benzil. ${ }^{13}$

SDR bulk fill has a higher fracture resistance (669.8 N) when compared to packable composite $(613,9 \mathrm{~N})$. This is similar to a study conducted by Atiyah AH and Baban LM (2014) in an in vitro study that showed that SDR bulk fill and packable composite did not have significant difference in their fracture resistance.

\section{CONCLUSION}

The effect of bulk fill composite on class I restoration of endodontically treated premolar teeth towards fracture resistance was significant $(\mathrm{p}<0.05)$. Bulk fill composite can be used as a final restoration in endodontically treated premolars with class I cavity, as it can also preserve sound tooth structure which can increase fracture resistance of the tooth.

\section{REFERENCES}

[1] Soares PV, Filho PCS, Queiroz EC, et al. Fracture resistance and stress distribution in endodontically treated maxillary premolars restored with composite resin. J Prosthodont 2008;17(2):114-9.

[2] Valian A, Moravej-Salehi E, Geramy A, et al. Effect of extension and type of composite-restored class II cavities on biomechanical properties of teeth: a three dimensional finite element analysis. J Dent 2015;12(2):140-50.

[3] Kalburge V, Yakub SS, Kalburge J, et al. A comparative evaluation of fracture resistance of endodontically treated teeth, with variable marginal ridge thicknesses, restored with composite resin and composite resin reinforced with ribbond: an in vitro study. Indian J Dent Res 2013;24(2):193-8.

[4] Scotti N, Eruli C, Comba A, et al. Longevity of class 2 direct restorations in root-filled teeth: a retrospective clinical study. J Dent 2015;43(5):499-505.

[5] Rocca GT, Krejci I. Crown and post-free adhesive restorations for endodontically treated posterior teeth: from direct composite to endocrowns. Eur J Esthet Dent 2013;8(2):156-79.

[6] Kishen A. Mechanisms and risk factors for fracture predilection in endodontically treated teeth. Analyze and Detect Bone Loss, Endodontic Topics 2006;13(1):57-83.

[7] Albers HF. Tooth-colored restoratives principles and techniques. $9^{\text {th }}$ edn. London: BC Decker Inc, 2002:111224.

[8] Moosavi H, Zeynali M, Pour ZH. Fracture resistance of premolars restored by various types and placement techniques of resin composites. Int J Dent 2012;2012:5.

[9] Ruiz JL. Dental technique-restorations with resinbased, bulk fill composites. AEGIS Dental Network, Compendium, Institute of Esthetic Dentistry United State 2010;31(5):14-5.

[10] El-Damanhoury H, Platt J. Polymerization shrinkage stress kinetics and related properties of bulk-fill resin composites. Oper Dent 2014;39(4):374-82.

[11] Atiyah AH, Baban LM. Fracture resistance of endodontically treated premolars with extensive MOD cavities restored with different composite restorations. Journal of Baghdad College Dentistry 2014;26(1):7-15.

[12] Hatrick CD, Eakle WS. Dental materials clinical applications for dental assistants and dental hygienists. $3^{\text {rd }}$ edn. Missouri: Elsevier, 2016:70-1.

[13] Dionysopoulos D. Bulk fill composite resins. A novelty in resin-based restorative materials. ARC Journal of Dental Science 2016;1(2):1-3. 